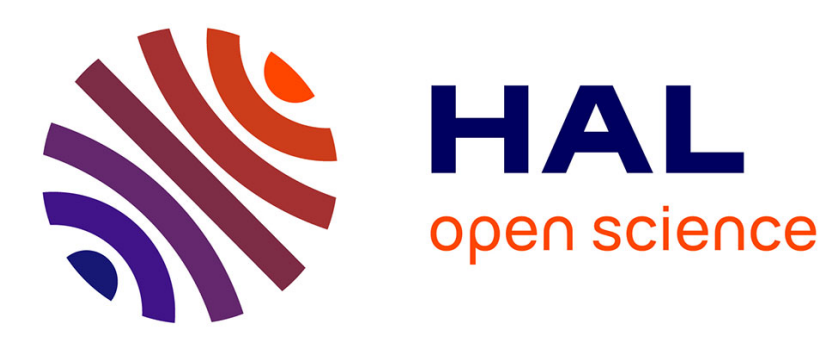

\title{
A modular CDF approach for the approximation of percentiles
}

Kingshuk Roy Choudhury, Sabin Tabirca

\section{To cite this version:}

Kingshuk Roy Choudhury, Sabin Tabirca. A modular CDF approach for the approximation of percentiles. Communications in Statistics - Simulation and Computation, 2008, 37 (10), pp.1948-1965. 10.1080/03610910802296356 . hal-00514329

\section{HAL Id: hal-00514329 \\ https://hal.science/hal-00514329}

Submitted on 2 Sep 2010

HAL is a multi-disciplinary open access archive for the deposit and dissemination of scientific research documents, whether they are published or not. The documents may come from teaching and research institutions in France or abroad, or from public or private research centers.
L'archive ouverte pluridisciplinaire HAL, est destinée au dépôt et à la diffusion de documents scientifiques de niveau recherche, publiés ou non, émanant des établissements d'enseignement et de recherche français ou étrangers, des laboratoires publics ou privés. 


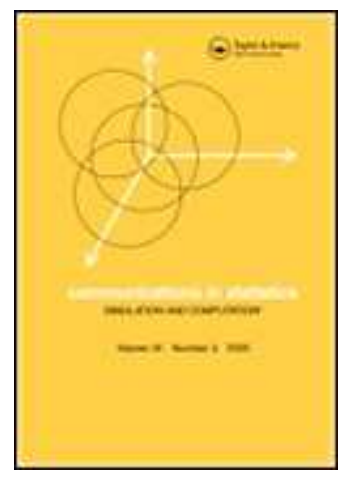

\section{A modular CDF approach for the approximation of percentiles}

\begin{tabular}{|c|c|}
\hline Journal: & Communications in Statistics - Simulation and Computation \\
\hline Manuscript ID: & LSSP-2007-0070.R1 \\
\hline Manuscript Type: & Original Paper \\
\hline $\begin{array}{r}\text { Date Submitted by the } \\
\text { Author: }\end{array}$ & $22-O c t-2007$ \\
\hline Complete List of Authors: & $\begin{array}{l}\text { Roy Choudhury, Kingshuk; University College Cork, Statistics } \\
\text { Department } \\
\text { Tabirca, Sabin; University College Cork, Computer Science }\end{array}$ \\
\hline Keywords: & massive data sets, approximate CDF, von Mises functional \\
\hline Abstract: & $\begin{array}{l}\text { This paper describes a method for computing approximate statistics } \\
\text { for large data sets. Such situations arise in applications such as } \\
\text { climatology, data mining and information retrieval. A modular } \\
\text { approximation to the CDF enables the reduction of a potentially } \\
\text { overwhelming computational exercise into smaller, manageable } \\
\text { modules. We illustrate the properties of this algorithm using a } \\
\text { simulated data set. It is shown that the maximum error between } \\
\text { the approximate CDF and actual CDF is never more than } 1 \% \text {. Under } \\
\text { smoothness assumptions, the expected approximation error is } \\
\text { much lower. Bounds for the approximation error of percentiles are } \\
\text { also derived. }\end{array}$ \\
\hline
\end{tabular}

\section{(s) ScholaroNE \\ Manuscript Central}




\begin{abstract}
:
This paper describes a method for computing approximate statistics for large data sets, when exact computations may not be feasible. Such situations arise in applications such as climatology, data mining and information retrieval(search engines). The key to our approach is a modular approximation to the CDF of the data. Approximate percentiles (as well as many other statistics) can be computed from this approximate CDF. This enables the reduction of a potentially overwhelming computational exercise into smaller, manageable modules. We illustrate the properties of this algorithm using a simulated data set. We also examine the approximation characteristics of the approximate percentiles, using a von Mises functional type approach. In particular, it is shown that the maximum error between the approximate CDF and the actual CDF of the data is never more than 1\% (or any other preset level). We also show that under assumptions of underlying smoothness of the CDF, the approximation error is much lower in an expected sense. Finally, we derive bounds for the approximation error of the percentiles themselves. Simulation experiments show that these bounds can be quite tight in certain circumstances.

keywords : modular approximation, approximate CDF, von Mises functional, approximation error
\end{abstract}

\title{
1. Introduction
}

The advent of fast computers has led to a virtual revolution in statistical methodology. Procedures that would have been unthinkable a decade or two ago are now possible due to the relentless increase in processing power. This increase in processing power can be attributed primarily to increased clock speeds of the CPUs and volatile memory (RAM) capacity, which have increased roughly as a square root of time, the so-called Moore's Law. However, the increase in computing power has been paralleled by the 
increasing size and complexity of data sets. Media such as magnetic tape allow us to store virtually unlimited quantities of data.

One particular field that deals with very large data sets is climatology. In climatology, data on various physical quantities, such as temperature, wind speed, rainfall, humidity etc. are available at potentially high resolution both spatially and temporally, over the past few decades. In part, this is due to the advent of various remote sensing devices, such as weather satellites. Apart from actual observations, many climatological data sets are actually the output of a modelling exercise, sometimes referred to as 'hindcasts'. Hindcasts 'generate' data by running climate models. An example of hindcasts on the global scale is the NCEP/NCAR model [Kalnay et al., 1996], which generates data on a $2.5^{\circ}$ x 6 hour grid. Data on finer resolution has also been generated using the High Resolution Limited Area Models (HIRLAM) (Sass et al.) which generates data on a $0.5^{\circ} \times 1$ hour grid. The size of such datasets is typically on the order of a few gigabytes (HIRLAM), to many terabytes, such as those in the NCAR database (http://www.scd.ucar.edu/news/03/features/0227.petabyte.html).

Datasets of this size pose unprecedented computational problems. In this paper, we look at the problems of computing statistics for such large data sets. In section 2 , we see that for certain statistics, such as moments, large data sets do not pose a big problem because of the additive structure of such statistics. However, for other statistics, particularly those based on ranks, we show that this no longer applies: so much so that it might be impossible to compute such statistics for very large data sets. In this paper, we propose a general methodology for computing an approximation to such statistics. This general solution relies on a 'modular' approximation to the empirical CDF (section 3). Many statistics can be written as functionals of the empirical CDF. Therefore if one can compute approximation to the CDF, one can compute an approximation to the statistic by applying the functional to this CDF. Moreover, the approximation error of the approximate statistic can easily be derived using a von Mises functional type analysis (section 4).

In this paper, we shall concentrate on the specific problem of computing percentiles of such data sets. The $p$-th percentile is defined as the data value such that $p$-percent of the data are less than it. An important use 
A conceptually simple method of computing the $p$-th percentile at each grid point would be to read all the data into a single 3 dimensional array, index by latitude, longitude and time. One could then sort the temporal data at each spatial location to obtain the percentiles. Unfortunately, it is not possible to create and process an array of $28 \mathrm{~Gb}$ on most current computers. An alternative approach is to do the processing for each grid point separately. Although this is conceptually feasible, [Karsten et al., 2005] have estimated that the computation would take approximately 1633 days on a single PIII, $1.2 \mathrm{~Gb}$ processor with $512 \mathrm{~Kb}$ Cache and $1 \mathrm{~Gb}$ RAM. The main problem is that the computation requires multiple opening and closing of each file, i.e. many I/O operations. The speed of these operations is constrained by the size of cache memory.

The solution presented by [Karsten et al., 2005] to this problem was to develop a parallel processing algorithm, which they ran on a 100 node cluster. The algorithm used the combined cache memory and RAM of the cluster to considerably reduce the I/O time. Using this algorithm, the compute time was reduced to as low as 52 minutes. In general, however, the availability of large parallel processing clusters is limited. Moreover, when datasets become larger, constraints on cache memory will hit even large parallel processing clusters. For instance [Pohl, 1969] has demonstrated that the mimimum number of elements required to compute a median in one pass is $n / 2$, where $n$ is the size of the dataset. To avoid such 
computational bottlenecks, [Karsten et al., 2005] have suggested an approximation scheme where the overall percentiles are approximated as averages of the block wise percentiles. The problem with this approach is that there are no guarantees as to how close the approximate percentile will be to the approximate percentile. In section 2, we exhibit a counter-example which demonstrates that the resulting approximation can be quite poor under certain circumstances.

One approach to avoiding such problems is by computing the approximate percentile based on a random sample. A randomised algorithm for computing approximate percentiles was proposed in [Cochran, 1977]. The basic idea here is to draw a random sample from the entire dataset and use the law of large numbers to get an approximate percentile. However, the problem with this approach is that we require the sample to be 'representative' of the dataset. [Cochran, 1977] addressed this problem using a stratification of the population. Once the strata are identified, random sampling within each stratum is used to produce a representative sample for the entire dataset. In climatology, however, random sampling can be problematic, because data are typically correlated. As the correlation structure is typically unknown a priori, random sampling can be difficult to achieve.

The computation of approximate percentiles has also received attention in the database literature. It has applications in areas such as selection, data mining and context based information retrieval. A number of different algorithms for computing approximate percentiles have been proposed in the database literatures over last three of decades [Schemiser and Deutsch, 1977; Munro and Paterson, 1980; Jain and Chlamtac, 1985; Guradraja and Srivastava, 1990; Agrawal and Swami, 1995; Alsabti et al., 1997; Manku et al. 1998 ]. A review of these algorithms can be found in [Alsabti et al., 1997 and Manku et al. 1998]. Some of the desirable features of these algorithms are:

i) repeatable: they will return the same answer on each data set if the calculation is repeated

ii) one-pass: the original data files need to be accessed only once

iii) have settable approximation bounds: the error in approximation can be made as accurate as desired (with a concomitant change in memory/computation requirements) 
iv) low main memory requirements: at any time, the algorithm only deals with buffers whose size is a fraction of $n$.

\begin{abstract}
Many of the algorithms mentioned above take a 'modular' approach to the problem of computation of approximate percentiles. In this approach, each file (or block) has to be opened (and processed) only once. The output from each block is later combined to get the final answer. One attractive feature of 'modular' computing is scalability. Scalability means that even a very large data set can be analysed in reasonable time given a large number of computers or processors, each of which may not be very powerful individually. Another advantage is that the order of processing the blocks is not important. In particular, it does not have to be simultaneous, unlike a parallel processing algorithm.
\end{abstract}

\begin{abstract}
Although the basic requirements for both the climatology and database sorting problems are similar, there is a marked difference in emphasis due to the application background. As climatology is a scientific discipline, there is a greater emphasis on the quantification of error [Kalnay et al., 1996]. This is because the statistics may be used for subsequent modelling, for instance as a time series [Lozano et al, 2004]. In such an analysis, it is important to quantify the 'error' in each observation (statistic). In section 4, we shall present some simple methods to quantify the approximation error and how it can be reduced under certain circumstances. Conversely, it is also true that such statistics may not need to be computed as often as in a database application, such as a search engine. In such applications, there will be a greater emphasis on computational issues, as in [Manku et al., 1998].
\end{abstract}

\title{
2. Averaging of blockwise percentiles
}

Some statistics, such as moments and frequencies, are naturally modular. For instance, to compute the mean $\bar{x}$ of a modular data set, all we need to compute are the means $\bar{x}_{i}$ and sizes $n_{i}$ of each block. One can then compute the overall mean as: 
[Karsten et al., 2005] have suggested the averaging of blockwise percentiles as a method for approximate computation of the overall percentile. Unfortunately, the computation of percentiles is not inherently modular. To see why this is the case, we consider a computer generated data set. The data set is generated as a random sample of size 100,000 from an exponential distribution with mean 1 . All computations done in this paper are done using the $\mathrm{R}$ statistical software package (cran.r-project.org). A histogram giving the distribution of this dataset is shown in fig 1. For the sake of definiteness, suppose we are interested in finding out the 95-th percentile of this dataset. The 95-th percentile of this dataset turns out to be 3.01 upto 2 significant decimals.

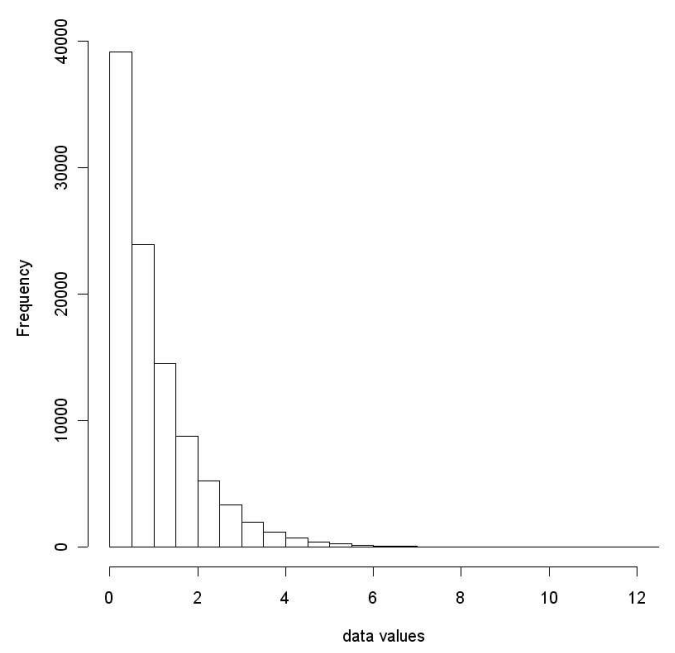

\section{Figure 1: Histogram of entire dataset}

Now we will divide our data set into two blocks of equal size. In the first instance, we will do the division in an equitable manner. We do this by randomly assigning half the data values to the first block and rest to the second block. The distribution of data in the resulting blocks is shown in fig 2. As we can see, the histograms in fig. 2 look very similar to each other. They are als o similar in shape to figure 1. In fact, 
when we compute the 95 -th percentile of each block, it turns out to be 3.01 in both cases. So, if we take the average of these two percentiles, we end up with a value (also 3.01) which is very close to the 95-th percentile of the entire data set.

In our second division, we divide in a more extreme manner. We do this by sorting the data in increasing order. We then assign the lowest $50 \%$ values to the first block and the highest $50 \%$ values to the $2^{\text {nd }}$ block. The distribution of data in the resulting blocks is shown in fig 3. As we can see, the histograms of the blocks in fig. 3 look quite different from each other. When we compute the 95-th percentile of each block, it turns out to be 0.65 for the $1^{\text {st }}$ block and 3.71 for the $2^{\text {nd }}$ block. Clearly there is a big difference between the 95-th percentiles of the two blocks, as we would expect. Moreover, when we compute an average of these two percentiles, we get a value of 2.18, which is quite far from the true value of 3.01 .

Thus the accuracy of the average of block wise percentiles as an estimator of the overall percentile depends heavily on the relative distributions of the data in the blocks.

1st block

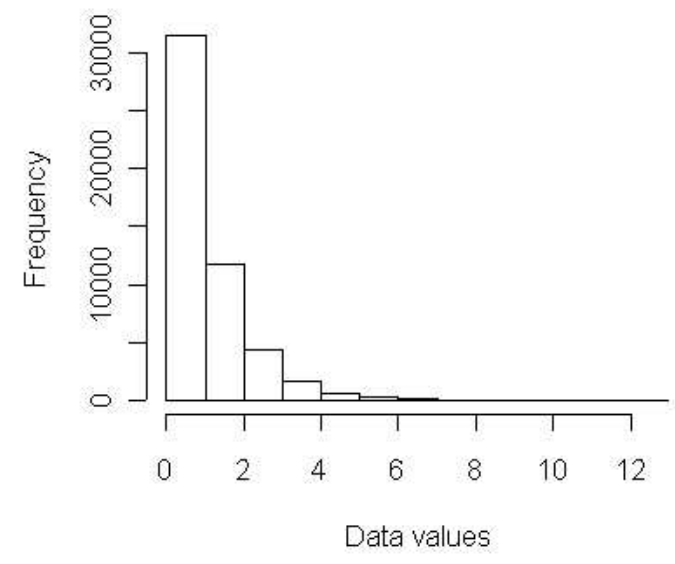

2nd block

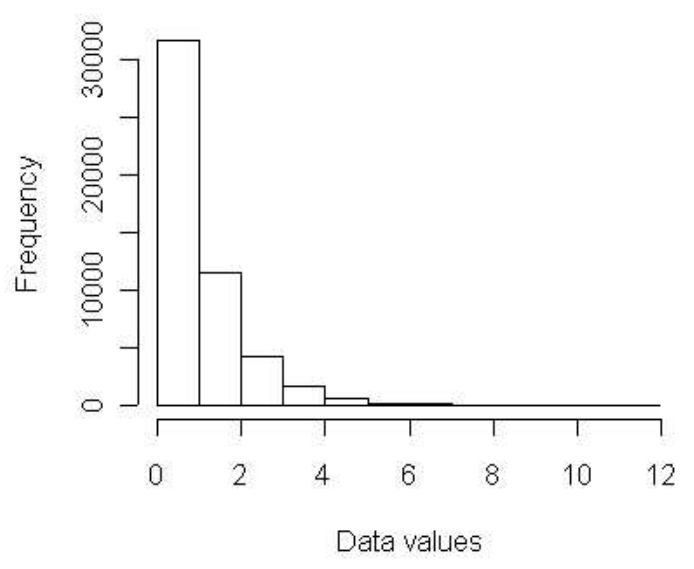

Figure 2: Histogram of blocks formed by random assignment. 
1st block

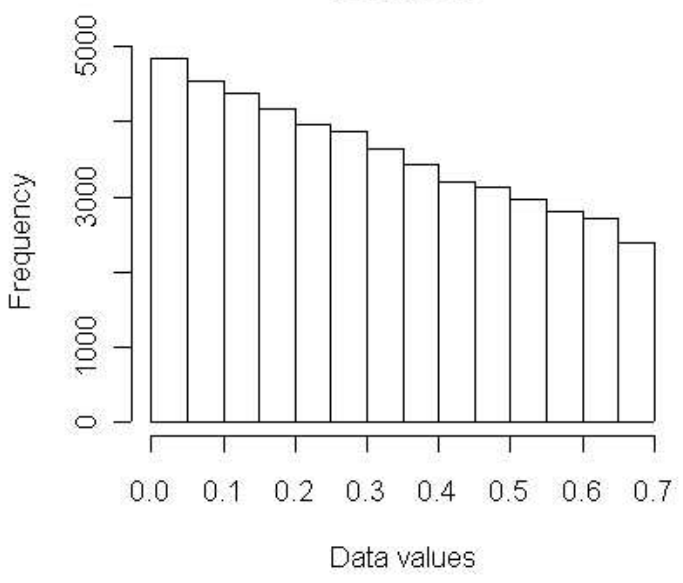

2nd block

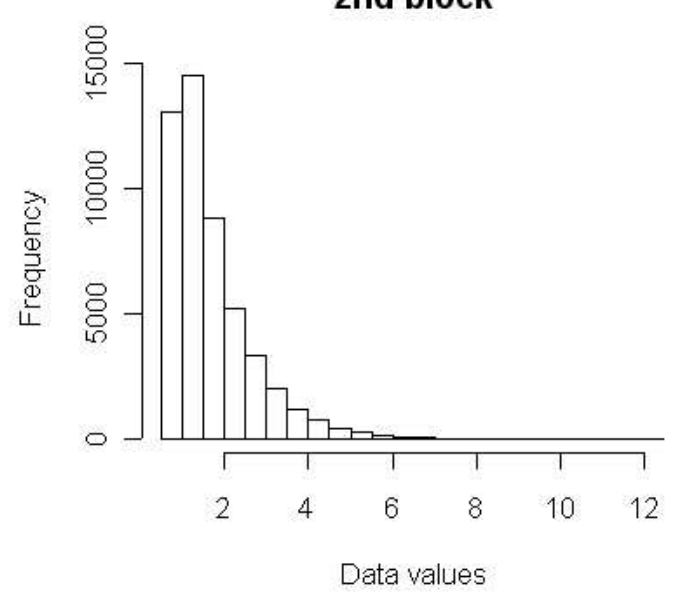

Figure 3: Histogram of blocks formed by sorted assignment.

In the situation where the distributions of the two blocks are identical, such as in fig. 2 , the average of the block wise percentiles will be the same as the overall percentile. However, when the block wise distributions are not identical, the average of percentiles will not in general give rise to the overall percentile. Even if we wanted to devise a weighted combination of the two block wise estimates, such a scheme would require knowledge of the relative juxtaposition of the two block wise distributions. The identical block wise distribution scenario will occur if distribution of data is stationary across blocks. If on the other hand, there is non-stationarity, for instance due to spatial or temporal effects, we will be in the situation where the block wise distributions are not identical. Even if the phenomena are not non-stationary, but periodic, the blocks could be in different phases leading to different block wise distributions.

Although the above discussion was in the context of two blocks, it is relatively straightforward to extend these arguments to the multi-block case. In practice, it is not possible to determine beforehand if we are in a stationary or non-stationary situation. Therefore it is safest to assume that we are in the setting of nonidentical block wise distributions. As we have seen, no pre-determined averaging scheme will give accurate estimates of the overall percentile in this case. Instead, as suggested in the above discussion, we will have to look at ways of estimating the distribution of the block wise data and their relative juxtaposition. 


\section{Modular Estimation of the CDF}

A standard approach to describe the probability distribution of a random variable $X$ is via its cumulative distribution function (CDF), usually denoted by $F(x)$. It is defined as:

$$
F(x)=\mathrm{P}(X \leq x)
$$

With this definition, the $p$-th percentile $x_{p}$ can also be viewed as solutions of the equation $F\left(x_{p}\right)=p$.

Thus there is a close connection between percentiles and the CDF. Given data, we can get an estimate of $F$, say $\hat{F}$. Clearly, the accuracy of the percentiles computed using this estimated CDF will depend on how close $\hat{F}$ is to $F$. A commonly used estimator for $F$ is the empirical CDF $F_{n}$, which is defined as:

$$
F_{n}(x)=\frac{\#\left(x_{i} \leq x\right)}{n}
$$

Here $n$ is the sample size and $x_{1}, x_{2}, \ldots, x_{n}$ are the data-points. As $n$ increases, the Glivenko-Cantelli lemma [ref . here] demonstrates that $\sup _{x}\left|F_{n}(x)-F(x)\right| \rightarrow 0$. Thus using $F_{n}$ instead of $F$ can yield reasonably accurate percentiles if $n$ is large.

The advantage of estimating $F_{n}$ instead of the percentiles directly is this can be computed in a modular fashion. We assume that our entire data vector $X$ is partitioned into $k$ blocks, each block having $n_{i}$ data points. Of course, $\sum_{i=1}^{k} n_{i}=n$, i.e.

$$
X=\left[X_{1}: X_{2}: \ldots: X_{\mathrm{k}}\right]
$$

It is easy to see that for any value $x$ :

$$
\#(X \leq x)=\#\left(X_{1} \leq x\right)+\#\left(X_{2} \leq x\right)+\ldots+\#\left(X_{k} \leq x\right)(3.4)
$$

Let $F^{i}$ denote the empirical CDF of the $i$-th block. Using equations (3.2) and (3.4), the empirical cdf can be written in a modular fashion: 


$$
F_{n}(x)=\sum_{i=1}^{k} \frac{n_{i}}{n} F^{i}(x)
$$

Although equation (3.5) does express the empirical CDF in modular fashion, it does not provide a practical method of computation. This is because we may need $2 n_{i}$ elements to store $F^{i}$. Therefore to compute $F_{n}$ from the $F^{i}$ we will need to handle $\mathrm{O}(n)$ elements, which brings us back to square one. Instead, we will compute compact approximations to the $F^{i}$, which we call $\hat{F}^{i}$ and use these to approximate $F_{n}$. Thus $\hat{F}_{n}$, our modular estimate of the CDF will be of the form:

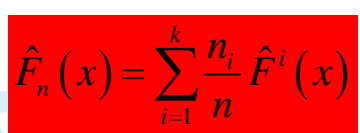

The formula in equation Error! Reference source not found. can also be re-written as follows:

$$
\hat{F}_{n}(x)=\frac{n_{-k}}{n} \hat{F}^{(k-1)}(x)+\frac{n_{k}}{n} \hat{F}^{k}(x)
$$

Where $\hat{F}^{(k-1)}(x)$ denotes the approximation based on the first $(k-1)$ blocks and $n_{-k}$ denotes the number of observations in these blocks. It can be seen that equation (3.7) is essentially a recursive relation, which means that it can be used as the basis for a sequential algorithm.

The importance of this result lies in the fact that the empirical CDF is equivalent to the order statistics of any dataset and therefore acts as a sufficient statistics for every dataset. This means that every measurable statistic is a function of the empirical CDF. Some can be explicitly written as a functional $\phi\left(F_{n}\right)$ of the empirical CDF, also known as von Mises functional [Serfling, 1988]. Using the modular approximation to the empirical CDF given in equation Error! Reference source not found. , one can construct a modular approximation to the statistic as :

$$
\hat{\phi}\left(F_{n}\right)=\phi\left(\hat{F}_{n}\right)
$$

In particular, the $p$-th percentile $x_{p}$ can be obtained using the functional $\phi_{p}(G)=G^{-1}(p)$. Other examples of von Mises functionals include the trimmed mean, moments and U-statistics. If the underlying 'population' 
$\mathrm{CDF}$ is denoted by $F$, the corresponding value of the functional in the population is given as $\phi(F)$. The error between the approximate statistic and this population quantity is can be written as:

$$
\hat{\phi}\left(F_{n}\right)-\phi(F)=\hat{\phi}\left(F_{n}\right)-\phi\left(F_{n}\right)+\phi\left(F_{n}\right)-\phi(F)
$$

Thus the approximation error to the population quantity can be written as the sum of two terms, one of which is the approximation error within the data set and the second is the sampling variability (difference between exact sample statistic and population parameter). Assuming that the data consists of a random sample of the population, it is known that the empirical CDF will converge almost surely to the 'population' CDF $F$. If in addition, it is known that the function is $\phi$ 'smooth', i.e. differentiable in a certain functional sense upto second order, [Serfling, 1988] proves that the sampling variability, i.e. the term $\phi\left(F_{n}\right)-\phi(F)$, will converge almost surely to zero as sample size goes to infinity. This is indeed the case with very large data sets. Therefore, for such data sets, we need only be concerned with the approximation error within a data set. In the rest of the paper, therefore, we will ignore the problem of sampling variability.

It should be noted that there are many statistics, which while not expressible as von Mises functionals of the empirical CDF, can be defined via the empirical CDF in an implicit manner. Examples of such statistics include M-, L- and R- statistics, as defined in [Serfling, 1988]. The analysis of these estimates is not pursued further in this paper.

\subsection{Computation of blockwise approximations}

Equation Error! Reference source not found.presents a general paradigm for computing modular approximations of the CDF. In order to compute the overall approximation, we first need to compute the blockwise approximations $\hat{F}^{i}(x)$. To compute these blockwise approximations we need to find the percentiles (or generally the quantiles) of the blocks. A number of computationally efficient algorithms have appeared in the literature [Munro and Paterson, 1980; Jain and Chlamtac, 1985; Agrawal and Swami, 1995; Alsabti et al., 1997; Manku et al. 1998]. The choice of which algorithm to use depends on factors like the number and size of blocks and the computational memory available. [Manku et al. 1998] have done a comparative simulation study to 
compare the performance of some of these algorithms. For the sake of completeness, we describe below a simple implementation of one of these algorithms [Alsabti et al., 1997]:

For block $i$, we compute its percentiles. To compute these we first need to sort the data in this block. Let $x_{[1]}^{i}, x_{[2]}^{i}, \ldots, x_{\left[n_{i}\right]}^{i}$ denote the data from $X_{1}$ sorted in increasing order. The percentiles are 101 numbers which can be obtained as follows

$$
\begin{aligned}
& x_{0.00}^{i}=x_{[1]}^{i} \\
& x_{0.01}^{i}=x_{\left[0.01 n_{1}\right]}^{i} \\
& x_{0.02}^{i}=x_{\left[0.02 n_{1}\right]}^{i} \\
& \ldots \\
& x_{0.0 j}^{i}=x_{\left[0.0 j n_{1}\right]}^{i} \\
& \ldots \ldots \\
& x_{1.0}^{i}=x_{\left[n_{1}\right]}^{i}
\end{aligned}
$$

The convention used here is that if $0.0 j n_{i}$ is not an integer, $x_{0.0 j}$ can be computed as an average of the two nearest ordered values of $x$. Using these percentiles, we can compute an estimator of the CDF based on $X_{1}$ alone, as follows:

$$
\begin{aligned}
\hat{F}^{i}(x) & =0 \quad \text { if } x \leq x_{[1]}^{i} \\
& =\frac{j}{n_{1}} \text { if } x_{[j]}^{i}<x \leq x_{[j+1]}^{i} \text { for } j=1,2, \ldots,\left(n_{i}-1\right)(3.10) \\
& =1 \text { if } x_{\left[n_{i}\right]}^{i}<x
\end{aligned}
$$

To store $\hat{F}^{i}$ we need to store only 202 numbers, the percentiles and the value of $\hat{F}^{i}$ at the percentiles. By definition, $\hat{F}^{i}=F^{i}$ at each of the percentiles. Therefore one can say that:

$$
\sup _{x}\left|\hat{F}^{i}(x)-F^{i}(x)\right| \leq 0.01(3.11)
$$

Of course, one can get more accurate estimates by storing more points. 


\subsection{Computation of the overall approximate CDF}

To compute $\hat{F}_{n}$ in equation Error! Reference source not found., we need to compute a weighted sum of the $k$ step functions $\hat{F}^{i}, i=1,2, . . k$. The problem in this computation is that the steps of the functions $\hat{F}^{i}$ are not synchronised. Potentially, therefore $\hat{F}_{n}$ could have as many as $101 k$ steps. The actual number of steps equals the number of unique points in the combined array $\left\{\left(x_{[1]}^{i}, x_{[2]}^{i}, \ldots, x_{\left[n_{i}\right]}^{i}\right), i=1,2, \ldots, k\right\}$. In practice, the sum in equation Error! Reference source not found. is computed by evaluating $\hat{F}^{i}$ at all of these unique points. This converts into the $\hat{F}^{i}$ into synchronised arrays of equal length, suitable for element wise addition.

(a)

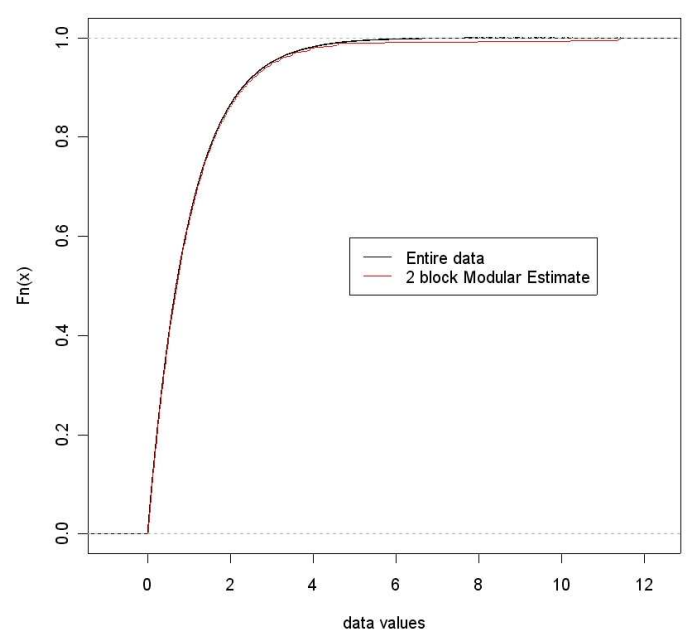

(b)

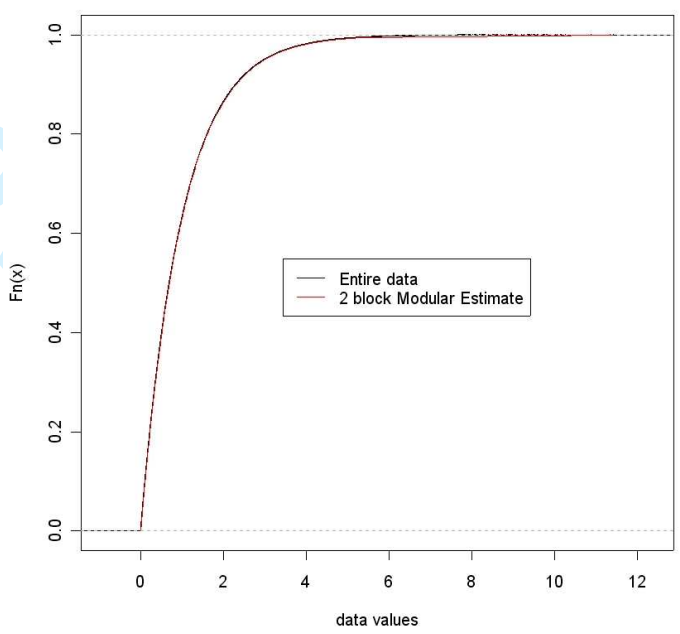

Figure 4: Comparison of empirical CDF (based on entire data) with two block modular approximation for data split with (a) random assignment and (b) sorted assignment.

\subsection{Examples of modular approximation}


We consider the examples presented in figs. 2 and 3. Both these figures relate to the same entire data set displayed in fig. 1. However, the data is split differently in either case. For fig. 2, the split is by random assignment to the two blocks, whereas for fig.3, the assignment is by prior sorting. The quality of the approximation given in equation Error! Reference source not found. is demonstrated in fig 4 (2 block split) and 5 (10 block split). For both 4(a) and (b) the modular approximation appears to be very close to the empirical CDF, which it is supposed to approximate. Discrepancies are only visually apparent at data values greater than 5 . This is a region where less than $1 \%$ of the entire dataset is concentrated. Incidentally, the $95^{\text {th }}$ percentile for both figure 4(a) and 4(b) is approximately 2.99 , which is quite close to the true value of 3.01. If anything, at the upper extremes of the data the quality of the approximation appears to be slightly better in fig 4(b) than in fig 4(a),. We will comment upon this phenomenon in the error analysis section.

(a)

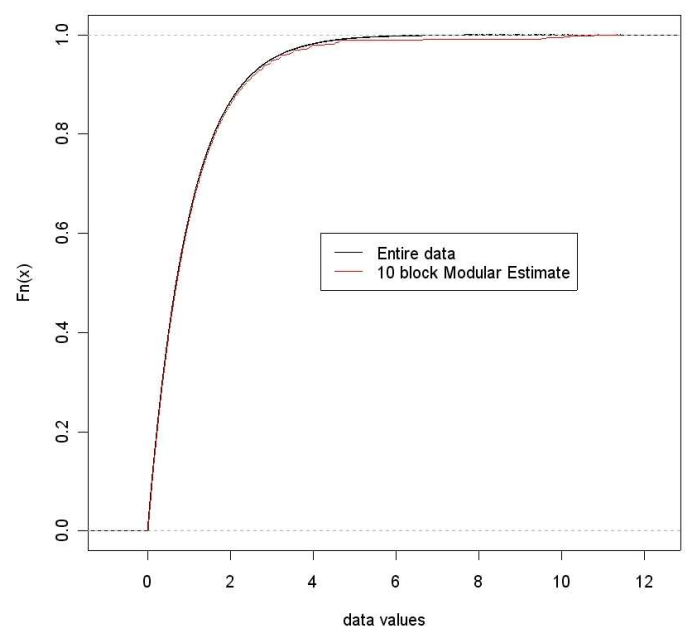

(b)

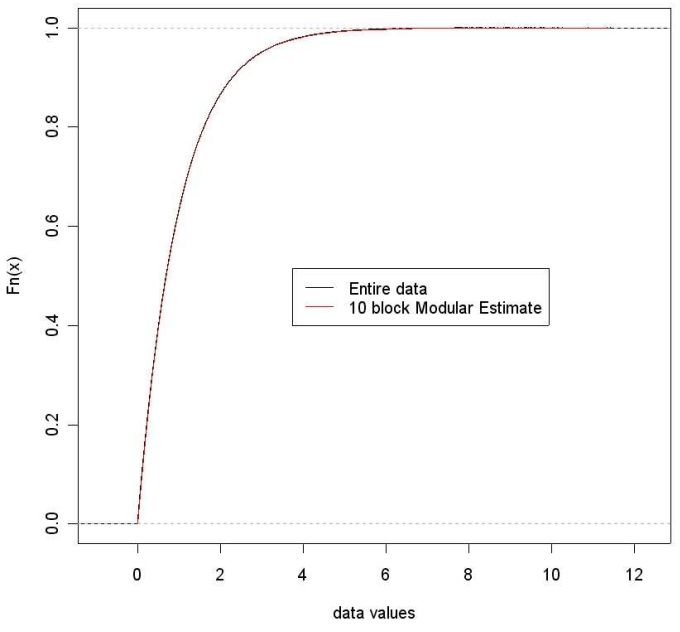

Figure 5 Comparison of empirical CDF (based on entire data) with ten block modular approximation for data split with (a) random assignment and (b) sorted assignment.

In figure 5 consider the modular approximation obtained when the same original data set is split into ten blocks, respectively by (a) random assignment (b) sorted assignment. Again, both approximations look 
very close to the original empirical CDF. This time, the approximate 95-th percentile are for (a) 3.05 (b) 2.97. It is slightly worse than the two block approximations, but not too much worse.

\section{Bounds for the approximation error}

\subsection{Bounds for the maximum error}

The goal of modular approximation $\hat{F}_{n}$ is to get as close to the empirical CDF, $F_{n}$, as possible. Therefore for error analysis, we need to consider the difference between these two functions. By equations (3.5) and Error! Reference source not found., we can write this difference as:

$$
F_{n}(x)-\hat{F}_{n}(x)=\sum_{i=1}^{k} \frac{n_{i}}{n}\left(F^{i}(x)-\hat{F}^{i}(x)\right)
$$

Applying the triangle inequality and taking supremum on both sides, we get the result that:

$$
\sup _{x}\left|F_{n}(x)-\hat{F}_{n}(x)\right| \leq \sum_{i=1}^{k} \frac{n_{i}}{n} \sup _{x}\left|F^{i}(x)-\hat{F}^{i}(x)\right|
$$

If we now use the approximation property of the $\hat{F}^{i}$ as given in equation(3.11), namely that the block wise approximations are accurate to the nearest percent, we get the result that:

$$
\sup _{x}\left|F_{n}(x)-\hat{F}_{n}(x)\right| \leq \sum_{i=1}^{k} \frac{n_{i}}{n} 0.01=0.01
$$

Equation (4.3) shows that given block wise estimates which are accurate to the nearest percent, we can construct a modular approximation which is also accurate to the nearest percent. This result is similar in effect to Lemmas 1, 2 and 3 of [Alsabti et al., 1997]. If a more accurate approximation of $F_{n}$ is required, it can be obtained by starting with more accurate block wise estimates of the $F^{i}$.

Of course, equation (4.3) is a worst case result. In many situations, the quality of the approximation will be much closer than $1 \%$. One such special case is when the data values in a certain range, say $\left[x_{a}, x_{b}\right]$ only occur in a few blocks, say $1,2, . ., l$, with $l<k$. We also assume that the range of data in the other blocks is 
either less than or greater than this range. This type of situation can occur in practice when we are look at 'extreme values', i.e. values that are unusually 'high' or 'low' and therefore have only occurred a few times in the entire data set, specially when such values tend to be clustered together. In this case blocks with data ranges above the range $[a, b]$ :

$$
F^{i}(x)=\hat{F}^{i}(x)=0 \text { for } x \in[a, b]
$$

and for blocks with data ranges above the range $\left[x_{a}, x_{b}\right]$ :

$$
F^{i}(x)=\hat{F}^{i}(x)=1 \text { for } x \in[a, b]
$$

If these values are substituted into equation (4.2), we get:

$$
\sup _{x \in[a, b]}\left|F_{n}(x)-\hat{F}_{n}(x)\right| \leq \sum_{i=1}^{k} \frac{n_{i}}{n} \sup _{x \in[a, b]}\left|F^{i}(x)-\hat{F}^{i}(x)\right| \leq 0.01 \sum_{i=1}^{l} \frac{n_{i}}{n}
$$

Thus, if the total size of the overlapping blocks is small, the maximum possible error in areas of nonoverlap will also be small. An extreme example of this sort of behaviour is manifested in the case when the data is divided into blocks by sorted assignment, such as in fig. 3. In this case, there is no overlap between the blocks. Another special case means that the minimum and maximum of the entire data set will always be correctly identified by the modular approximation.

\subsection{Bounds for average error}

The previous section looks at maximum errors. However, this may not give an overall picture of the approximation process. To get such a picture, we consider the average approximation error. We define the average approximation error to be the average of

the overall error, $\left(F_{n}(x)-\hat{F}_{n}(x)\right)$ over all possible values of $x$. For instance, if the overall distribution is supported between the values $\mathrm{L}$ and $\mathrm{U}$ (both finite), we can define the average error to be:

$$
a v\left|F_{n}(x)-\hat{F}_{n}(x)\right|=\frac{1}{U-L} \int_{L}^{u}\left|F_{n}(x)-\hat{F}_{n}(x)\right| d x
$$

If the support is infinite, we can extend the definition in equation (4.7) by a limiting argument. From equations (4.1) and (4.2) it is clear that the behaviour of the overall error is governed by the errors in the block wise estimates. For instance block wise estimates that produce low individual error will also produce 


\subsection{Reducing error by linear interpolation}

The results presented upto now are applicable to any dataset, i.e. possessing any underlying CDF. We now address the question as to whether improvements in the approximation error can be achieved for certain special classes of CDFs, namely those that are smooth of certain orders. In particular, we look at first order differentiable CDFs.

Given a differentiable CDF (i.e. a probability density function which is absolutely continuous with respect to Lebesgue measure), we can write down a Taylor series approximation for the value of the CDF at any given point $x$ in terms of the value at the next lowest percentile $x_{p}$ : 


$$
F^{i}(x)=F^{i}\left(x_{p}^{i}\right)+\left(x-x_{p}^{i}\right) F^{i}(\xi)
$$

Here, $F^{i}(\xi)$ is derivative of the CDF (p.d.f.) at an intermediate point $\xi$ between $x$ and $x_{p}^{i}$. By

recognizing that $F^{i}\left(x_{p}^{i}\right)=\hat{F}^{i}(x)$, we can see that the second term on the RHS of equation (4.10) gives us an expression for the error discussed in the previous subsection. Assuming for the moment that $F^{i}{ }^{\prime}(\xi)$ is a constant, this expression shows that the error is increasing linearly in $\left(x-x_{p}^{i}\right)$. The quantity $F^{i}(\xi)$ will be a constant if the CDF is that of a uniform random variable, because then it is linear. It is not constant in general. However, if $x_{p}^{i}$ and $x_{p+1}^{i}$ are not too far apart, it is not unreasonable to assume that $F^{i}$ is piecewise linear between the percentiles, in this case, we can write:

$$
F^{i}(\xi)=\frac{\left(F^{i}\left(x_{p}^{i}\right)-F^{i}\left(x_{p+1}^{i}\right)\right)}{\left(x_{p}^{i}-x_{p+1}^{i}\right)}
$$

Substituting this in equation (4.10), we get

$$
F^{i}(x)=F^{i}\left(x_{p}^{i}\right)+\left(x-x_{p}^{i}\right) \frac{\left(F^{i}\left(x_{p}^{i}\right)-F^{i}\left(x_{p+1}^{i}\right)\right)}{\left(x_{p}^{i}-x_{p+1}^{i}\right)}
$$

Equation is equivalent to obtaining $F^{i}(x)$ by linear interpolation given the values at the percentiles just above and below it. This gives an alternative piecewise linear estimate of $F^{i}(x)$ based on the percentiles:

$$
\hat{F}_{l}^{i}(x)=F^{i}\left(x_{p}^{i}\right)+\frac{\left(x-x_{p}^{i}\right)}{\left(x_{p+1}^{i}-x_{p}^{i}\right)}\left(F^{i}\left(x_{p}^{i}\right)-F^{i}\left(x_{p+1}^{i}\right)\right)
$$

This type of piecewise linear approximation of the CDF function is used in actuarial practice for the estimation of survival probabilities from life tables (Benjamin and Pollard). The nature of the approximation error in $\hat{F}_{l}^{i}(x)$, i.e. $\left(\hat{F}_{l}^{i}(x)-F^{i}(x)\right)$ depends on the underlying CDF. Two examples of 
the distribution of this error across $x$ values are given in fig. 6 . Although the shape of the distribution is different in fig. 6(a) and (b) there are some features in common, which distinguish it from the error in the piecewise constant approximation. Firstly, the shape of the distribution depends on the underlying CDF, whereas the distribution of errors in always uniform in the piecewise constant case.

Secondly, the error values can be both positive and negative. In fact the sum of these deviations is practically zero in both cases, indicating there is no significant systematic over or under estimation by the piecewise linear approximation. Thirdly the average (absolute) error, as defined by equation (4.7), turns out to be approximately 0.0002 for both fig $6(a)$ and (b). This is substantially less than the average error of 0.005, which holds for the piecewise constant approximation. Thus, according to the average measure, the component wise piecewise linear approximation appears to have significantly better approximation characteristics than the piecewise constant approximation. Using equation (3.5), we can then come up a piecewise linear approximation to the overall CDF ,as follows:

(a)

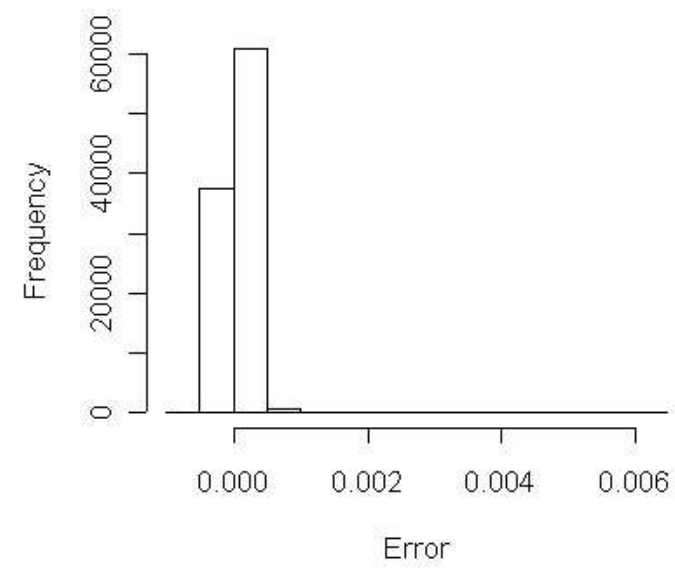

(b)

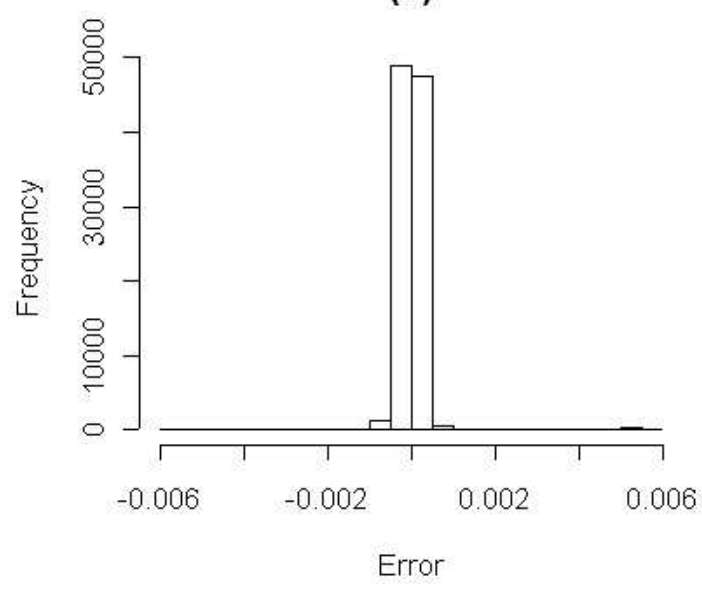

Figure 6: Distribution of error in linear approximation across data values, when the underlying CDF is (a) Exponential (b) Gaussian. The distribution was calculated from a random sample of size 100000 in each case.

$$
\hat{F}_{n}^{l}(x)=\sum_{i=1}^{k} \frac{n_{i}}{n} \hat{F}_{l}^{i}(x)
$$


Following equations (4.2) and (4.9), we can compute bounds for the supremum and average approximation errors which can be incurred with the piecewise linear approximation:

$$
\sup _{x}\left|F_{n}(x)-\hat{F}_{n}^{l}(x)\right| \leq \sum_{i=1}^{k} \frac{n_{i}}{n} \sup _{x}\left|F^{i}(x)-\hat{F}_{l}^{i}(x)\right|
$$

and

$$
a v\left|F_{n}(x)-\hat{F}_{n}^{l}(x)\right| \leq \sum_{i=1}^{k} \frac{n_{i}}{n} a v\left|F^{i}(x)-\hat{F}_{l}^{i}(x)\right|
$$

Note that the inequality is required in equation (4.16) because the approximation error is no longer nonnegative. The terms in the RHS of equations (4.15) and (4.16), namely $\sup _{x}\left|F^{i}(x)-\hat{F}_{l}^{i}(x)\right|$ and $a v\left|F^{i}(x)-\hat{F}_{l}^{i}(x)\right|$ can be computed while calculating $\hat{F}_{l}^{i}(x)$, i.e. at the modular stage, without too much extra computation involved.

\subsection{Bounds for approximation of percentiles}

The error analysis done so far has looked at the bounds for the error incurred in the approximation of $F_{n}$. However the goal of the paper is to approximate percentiles. We have noted that there is a close connection between the two approximations, because percentiles can be obtained as solutions to equation (3.1).In this subsection, we will compute error bounds for the approximate percentiles in terms of the error bounds for $F_{n}$. In order to do so, we must consider a Taylor series approximation of $\hat{F}_{n}^{l}(x)$. This is possible to calculate because it is a piecewise linear function, so derivatives exist at most points, except at the percentiles of the $\hat{F}_{l}^{i}(x)$.

$$
\hat{F}_{n}^{l}\left(x_{p}\right)=\hat{F}_{n}^{l}\left(\hat{x}_{p}\right)+\left(x_{p}-\hat{x}_{p}\right)\left(\hat{F}_{n}^{l}\right)^{\prime}(\xi)
$$


Here $x_{p}$ denotes the actual $p$-th percentile, $\hat{x}_{p}$ denotes the approximate $p$-th percentile obtained using the linear approximation and $\xi$ denotes a point in between these two. Using equation (4.14), we can write the derivative as:

$$
\left(\hat{F}_{n}^{l}\right)^{\prime}(\xi)=\sum_{i=1}^{k} \frac{n_{i}}{n}\left(\hat{F}_{l}^{i}\right)^{\prime}(\xi)
$$

The derivatives on the RHS of equation (4.18) can be computed using equation (4.11).

Using the property that $\hat{x}_{p}$ is the p-th percentile of $\hat{F}_{l}^{i}(x)$ and $x_{p}$ is the $p$-th percentile of $F_{n}$, we get from equation (4.17):

$$
\begin{aligned}
\hat{F}_{n}^{l}\left(x_{p}\right) & =p+\left(x_{p}-\hat{x}_{p}\right)\left(\hat{F}_{n}^{l}\right)^{\prime}(\xi) \\
& =F_{n}\left(x_{p}\right)+\left(x_{p}-\hat{x}_{p}\right)\left(\hat{F}_{n}^{l}\right)^{\prime}(\xi)
\end{aligned}
$$

Rearranging equation (4.19) allows us to write the approximation error of $\hat{x}_{p}$ in terms of the approximation error of $\hat{F}_{n}^{l}(x)$ :

$$
\left(x_{p}-\hat{x}_{p}\right)=\frac{\hat{F}_{n}^{l}\left(x_{p}\right)-F_{n}\left(x_{p}\right)}{\left(\hat{F}_{n}^{l}\right)^{\prime}(\xi)}
$$

Equation (4.20) shows us that the approximation error in $\hat{x}_{p}$ increases with the approximation error in $\hat{F}_{l}^{i}(x)$ and decreases with the value of the derivative $\left(\hat{F}_{n}^{l}\right)^{\prime}(\xi)$. A predicted upper bound for this approximation error can be computed using the bound in equation (4.15):

$$
\left|x_{p}-\hat{x}_{p}\right| \leq \frac{\sup _{x}\left|\hat{F}_{n}^{l}(x)-F_{n}(x)\right|}{\left(\hat{F}_{n}^{l}\right)^{\prime}(\xi)}
$$

An example of the effectiveness of these predicted upper bounds is shown in fig 7. In this figure, actual approximation errors (displayed by + symbols) are calculated by subtracting the actual percentiles of the data shown in fig. 1 from the approximate percentiles obtained using the linear approximation given in equation (4.14). These errors are plotted against their probabilities: for example the approximation error for the median (50-th percentile) is plotted against 0.5 . The black line shows the predicted upper bound 
calculated using a version of equation (4.21). The supremum in the numerator is replaced by 0.01 . The derivative is calculated as in equation (4.18), but is then smoothed to reduce the inherent variability in the piecewise constant derivative approximation. From these plots, we can see that the approximation errors are high for the higher percentiles. This is to be expected, because of the long tail in the distribution of the data, as shown in fig. 1.

In comparing fig 7 (a) and (b), we see that the errors in (b) are in general much lower than for (a). Also, the upper bound in fig. 7(a) appears to be much closer to the observed errors than for fig. 7(b). This is due to the fact that for 7(b), there is no overlap between the blocks. As explained in equation (4.6), the approximation error in the $\mathrm{CDF}$, which is the numerator in equation (4.21), is much lower. This leads to a distant upper bound when we use the crude upper bound of 0.01 for the approximation error in the CDF. On the other hand, there is complete overlap in the blocks in fig. 7(a), which makes 0.01 a much more reasonable upper bound for the approximation error in the CDF. This leads to a much tighter upper bound for the percentile error.

(a)

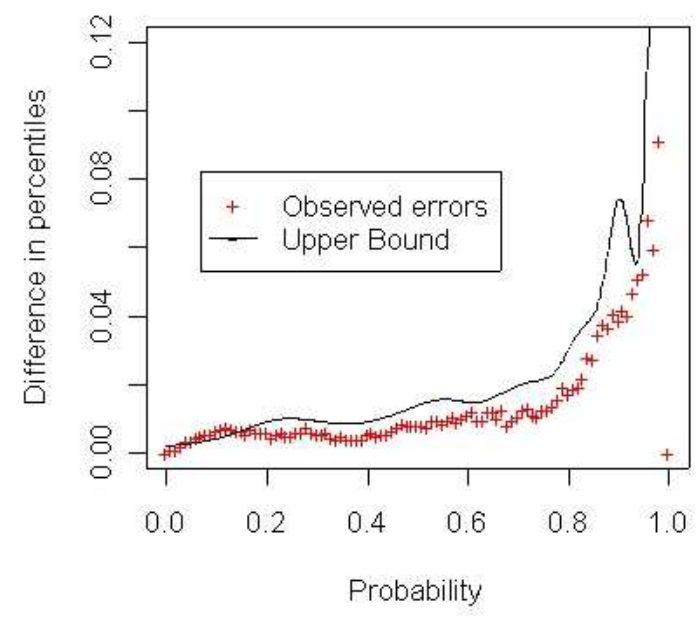

(b)

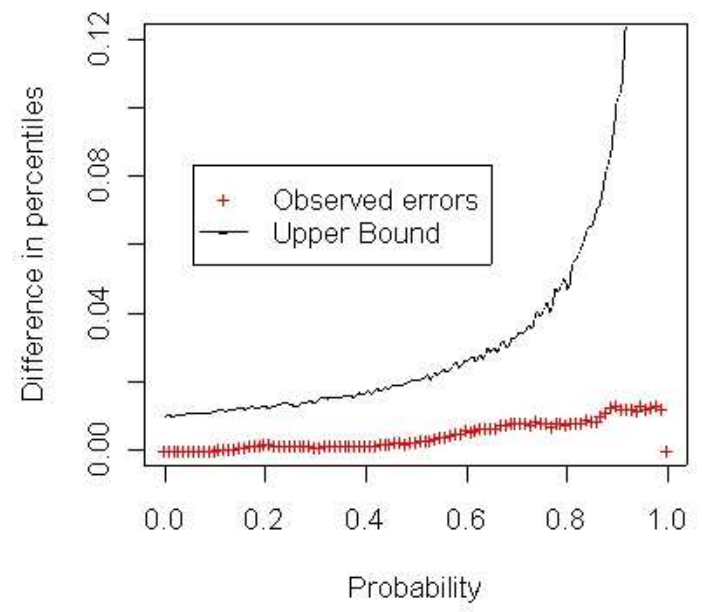

Figure 7: Errors in percentiles for the ten block modular approximations. Errors and the predicted upper bound are shown for (a) Blocks determined by random assignment (b) Blocks determined by sorted assignment 


\title{
5. Summary and Discussion
}

\begin{abstract}
The identity in equation (3.5) provides a basis for modular computation of the empirical CDF, which can then be used to compute percentiles and other statistics of a large data set. To reduce the storage requirements of this calculation, we have suggested an approximation to the empirical CDF, which requires the computation of the percentiles of each block of the data. It is also shown the approximation error of this procedure is at most $1 \%$. Bounds for the approximation error in the percentiles are also derived. We now discuss some of the limitations and possible extensions of the results presented here.
\end{abstract}

The presentation of the approximation method in this paper is motivated by the modular computational paradigm. In this setting, we assume that each block is processed by a separate machine (or processor) and the results are ultimately assimilated. However, the algorithms presented can easily be modified to suit a more limited setting. Using the same type of approximation as presented in section 3.1, we can compute a sequential approximation of the empirical CDF. In this setting, we assume that there is only a single processor at our disposal. Each block of data is processed sequentially and the approximate CDF is updated using equation (3.7). The final approximation will be identical to that obtained by the modular paradigm. The only difference will be in the computational implementation and the potential loss of time from not having processed the blocks simultaneously. It follows that the approximation errors will also be similar. In the context of the percentile calculation problem presented in [Karsten et al., 2005], which is also the briefly discussed in the introduction of this paper, both the sequential and modular computational approximations discussed here can create potentially large savings in computational space and time. This is because when using a traditional algorithm, [Karsten et al., 2005] noted that a substantial portion of the computational overhead required for this dataset arose due to the repeated opening and closing of the data block files. On the other hand, the modular, particularly the sequential algorithm requires each file to be accessed only once. 
One of the reassuring aspects of modular approximation is that the approximation error does not appear to significantly increase as the number of blocks increases. This is borne out in Fig 5, where the approximation error for the ten block approximation does not appear to be significantly worse than the two block approximation shown in Fig 4.This is true certainly in case of maximal error, where we have a global upper bound of $1 \%$ when we use percentiles for approximation. This upper bound applies no matter what the underlying data and no matter how many blocks are used. As noted in the text, this upper bound can be lowered to any desired level by increasing the number of percentiles that are recorded for each block. Of course, there is a concomitant increase in storage requirement, as well as some extra computation is required. A proper choice of the maximal allowable error can be made by balancing the size of the data and the processing capacity available. A sharper bound for the maximal error is provided in equation (4.6). In practice, the actual error will depend on the degree of periodicity, autocorrelation and nonstationarity present within and across blocks. In section 3.3, we have considered the least and most favourable scenarios for error (ordered allocation and random allocation respectively). In specific applications, it may be possible to get a better bound on the maximal error for the particular setting by making use of the data structure and/or some data analytic techniques.

In computational terms, the algorithm has a similar requirement to the algorithm presented in [Alsabti et al., 1997]. Using arguments presented in the latter paper, it can be shown that the order of computation is $\mathrm{O}(n$ $\log s$ ), where $\mathrm{s}$ is the number of quantiles being computed for each block ( $s=101$ in this paper). [Manku et al. 1998], have presented a much more sophisticated algorithm which claims to have lower computational overheads. Although such algorithms are meant to compute approximate percentiles, it should not be difficult to use these approximate percentiles to compute an approximate CDF as described in section 3.1.

A unique aspect of the analysis presented in this paper is the ability to provide error bounds for the percentiles themselves (section 4.4), rather than just the approximation probability. This may be of use if the approximate quantiles are going to be used for modelling purposes [Lozano et al. 2004], as then these bounds can be used as a basis for computation of standard errors of estimates. The brief simulation study shows that while these bounds can be quite tight in situations where there is a lot of overlap between the 


\title{
6. References:
}

\author{
Agrawal R.and Swami A., A One-Pass Space-E_cient Algorithm for Finding Quantiles, Proc. 7th Intl. \\ Conf. Management of Data (COMAD-95), Pune, India, 1995.
}

\begin{abstract}
K. Alsabti K., Ranka S., and Singh V., A One-Pass Algorithm for Accurately Estimating Quantiles for Disk-Resident Data", Proc. 23rd VLDB Conference, Athens, Greece, 1997.
\end{abstract}

Benjamin, B. and Pollard J., The analysis of mortality and other actuarial statistics, $3^{\text {rd }}$ edition, Institute of Actuaries and Faculty of Actuaries, 1993

Cochran.W., Sampling Techniques, Wiley, $3^{\text {rd }}$ edition, 1977.

Gunther, H.; Rosenthal, W.; Stawarz, M.; Carretero, J.C.; Gomez, M.; Lozano, I.; Serrano, O.; Reistad, M. The Wave Climate of the Northeast Atlantic Over the period 1955-1994: The WASA Wave Hindcast. The Global Atmsphere and Ocean System, 6, 121-163, 1998. 
Jain, R. and Chlamtac, I.,The P2 Algorithm for Dynamic Calculation for Quantiles and Histograms without Storing Observations, Comm. ACM, vol. 28, pp. 10761085, 1985.

Kalnay, E., Kanamitsu, M., Kistler, R., Collins, W., Deaven, D., Gandin, L., Iredell, M., Saha, S., White, G., Woollen, J., Zhu, Y., Chelliah, M., Ebisuzaki, W., Higgins, W., Janowiak, J., Mo, K.C., Ropelewski, C., Wang, J., Leetmaa, A., Reynolds, R., Jenne, R., Joseph, D. The NCEP/NCAR reanalysis project. Bulletin of the American Meteorological Society, 77, 431-471, 1996.

Karsten, S., Tabirca, S., Vijaykuman, N., Parallel Approach for the 90\%-tile Wind and Wave Hindcast over Irish and Celtic Seas, Proceedings of the Conference on Information Technologies in Environmental Engineering (ITEE'2005), Otto-von-Guericke-Universität Magdeburg, Germany, September 25 - 27, 2005

Lozano, I., Devoy, R. J. N., May, W. and Andersen, U. Storminess and vulnerability along the Atlantic coastlines of Europe: an analysis of storm records and of a greenhouse gases induced climate scenario. Marine Geology, 210, 205-225, 2004.

Manku, G., Rajagopalan, S. and Lindsay, B., Approximate Medians and other Quantiles in One Pass and with Limited Memory, Proc. ACM SIGMOD Conference, Seattle, 1998

Munro, J. and Paterson, M., Selection and Sorting with Limited Storage, Theoretical Computer Science, 12, 315-323, 1980.

Pohl, I., A minimum storage algorithm for computing the median, IBM Research Report RC2701 (\#12713), 1969

Ramsay, J., Monotone regression splines in action, Statistical Science, 3, 425-461, 1988

URL: http://mc.manuscriptcentral.com/Issp E-mail: comstat@univmail.cis.mcmaster.ca 
Sass, B.H.; Nielsen, N.W.; Jørgensen, J.U.; Amstrup, B.; Kmit, M. The Operational HIRLAM System at DMI. Danish Meteorological Institute Technical Report 00-26, 2000

Serfling, R., Approximation theorems of mathematical statistics, Wiley, 1980

Weisse, R., Gayer, G. An Approach Towards a 40-Year High-Resolution Wave Hindcast for the Southern North Sea. Proceedings of the $6^{\text {th }}$ International Workshop on Wave Hindcasting and Forecast, Monterey, USA, 2000. 